\title{
Digital Holographic Imaging the Future of Radiology: A Review Article
}

\author{
Mustafa Z. Mahmoud, $\mathrm{PhD}^{1 *}$; Hatem A. Alonazi, BSc${ }^{1}$; Moram A. Fagiry, $\mathrm{MSc}^{1}$; \\ Batil Alonazi, $\mathrm{PhD}^{1}$; Salem Saeed Alghamdi, $\mathrm{PhD}^{2}$; Rowa Aljondi, $\mathrm{PhD}^{2}$; \\ Mahasin G. Hassan, $\mathrm{PhD}^{3}$ \\ ${ }^{1}$ Radiology and Medical Imaging Department, College of Applied Medical Sciences, \\ Prince Sattam bin Abdulaziz University, Al-Kharj, Saudi Arabia \\ ${ }^{2}$ Department of Medical Imaging and Radiation Sciences, College of Applied Medical Sciences, \\ University of Jeddah, Jeddah, Saudi Arabia \\ ${ }^{3}$ Radiological Sciences Department, College of Health and Rehabilitation Sciences, \\ Princess Nourah bint Abdulrahman University, Riyadh, Saudi Arabia
}

\begin{abstract}
The potential that digital holographic imaging (DHI) holds in the future of radiology in $3 \mathrm{D}$ will undoubtedly lead to revolutionizing the guidance of minimally invasive surgical and percutaneous procedures, which are currently being performed using 2D and/or 3D images displayed on flat screens. This review will update the reader on the definition and processes of digital holography, holographic applications in radiology, limitations of holography and DHI and their significance in the future of radiology. Furthermore, the review elaborates upon the many open questions on how to exploit DHI fully in radiological practice.

(International Journal of Biomedicine. 2020;10(3):206-210.)
\end{abstract}

Key Words: augmented reality $\bullet$ digital holographic imaging $\bullet$ radiology $\bullet$ virtual reality

\section{Abbreviations}

2D, two dimensions; 3D, three dimensions; CT, computed tomography; DH, digital holography; DHI, digital holographic imaging; MRI, magnetic resonance imaging; PACS, picture archiving and communication system; PET, positron emission tomography.

\section{Introduction}

The term hologram, with "holo" meaning complete and "gram" meaning message, has long been used to refer to both the technique of wave-front reconstruction by interference, diffraction and reflection, and an image genre of a virtual encounter between an immaterial, usually 3D image of an absent human and living humans. (1) Holography, or "whole

*Corresponding author: Professor Mustafa Z. Mahmoud Radiology and Medical Imaging Department, College of Applied Medical Sciences, Prince Sattam bin Abdulaziz University, E-mail: m.alhassen@psau.edu.sa,PO Box: 422,Zip Code: 11942, Al-Kharj, Saudi Arabia. drawing," is a method by which an exact visual representation of an object in three physical dimensions can be created using laser beams, the properties of interference and diffraction, light intensity recording and the illumination of the recording. The images change according to the relative position of the individual viewer, as if the objects displayed are actually present. $^{(2)}$

Dennis Gabor, a Hungarian-British electrical engineer and physicist, was awarded the Nobel Prize in Physics for inventing holography in $1971 .^{(2)}$ Holograms, which have only been around since the latter part of the last century, enable the visual display of an object in 3D using light-physics. Digital holography (DH) has been implemented in many areas, including microscopy, and data storage and processing. ${ }^{(3-5)}$ The creation of holograms to aid 
medical and clinical decision-making has been discussed in the existing literature on the subject for over two decades. ${ }^{\left({ }^{6}\right)}$ Two types of holography are available: conventional and dynamic. Conventional holography represents static images of 3D objects, whereby a change in the viewer's angle of vision results in a change of perspective of the holographic object. Additionally, a dynamic holograph is one that adds motion to the $2 \mathrm{D}$ or $3 \mathrm{D}$ object. $^{(7)}$

Live X-ray and live 3D cardiac ultrasound imaging could be used simultaneously to guide minimally invasive structural heart repair procedures, with ultrasound images providing detailed insights into the heart's soft tissue anatomy, and with the X-ray imaging providing visualization of catheters and heart implants. Medical holography is actually the integration of all these modalities. However, 3D viewing technologies are not able to create truly accurate 3D renditions: They are, in actuality, 2D images gathered from MRI, CT scanner, ultrasound and other devices, stitched together to create a rough 3D image. Furthermore, it is extremely laborious and time-consuming to create $3 \mathrm{D}$ visualizations from these $2 \mathrm{D}$ images. This makes treatment and making diagnostic decisions based on these images rather challenging, and their accuracy is often questionable. Medical holography can be the answer to these challenges. By rendering these $2 \mathrm{D}$ images into $3 \mathrm{D}$ floating projections, this technology will revolutionize the way the medical professionals plan and carry out their treatments and surgeries: enabling physicians to zoom in and out of the images to get a better view of the areas under observation that are unclear in the initial scans. ${ }^{(1)}$ To date, the incorporation of technology in the medical field appears to lag behind, although promising advances in medical imaging have been witnessed in the fields of ultrasound, MRI, and PET. ${ }^{(8)}$ Bruckheimer et al.$^{(9)}$ have applied the DH technology to generate interactive, realtime, 3D holograms of cardiac imaging data based on rotational angiography and live 3D transoesophageal echocardiography. This new technology allows for the visualization of medical images in 3D without the need for 3D glasses. The operators can interact with the real-time dataset to crop, rotate, magnify, and move structures as they float in front of the operators, who will gain the advantage of appreciating the depth and proximity of adjacent anatomy with greater ease.

In the real world, the use of classical holographic technology has resulted in relatively primitive designs, and now we are witnesses to another major development in holographic technology. The True 3D Viewer, by EchoPixel, aims to solve this problem by providing medical professionals with a hologram of the organs, which can be moved around, zoomed in on or manipulated in actual 3D space. This new technology might not necessarily improve medical practices rapidly, but it can make diagnosing patients and treatment processes more effective. Furthermore, this 3D holography could avoid the use of physical procedures that may cause discomfort to patients and help detect problems in complex organs such as the heart or the brain..$^{(1)}$ The addition of motion represents a significant advancement over current 3D displays, which can only show 3D static images. It also opens the door to three major potential uses of holography in radiology: i) teaching anatomy, ii) PACS storage, and iii) displaying more complex anatomic images that can be used to present more informative reports to physicians and their patients. ${ }^{(10)}$

This review article will update the reader on the definition and processes of $\mathrm{DH}$, holographic applications in radiology, limitations of holography and DHI, and the crucial role this technology has in the future of radiology.

\section{Literature Review}

An inclusive literature review was conducted to update the reader on the definition and processes of $\mathrm{DH}$, holographic applications in radiology, limitations of holography and $\mathrm{DH}$ and their significance and impact with regard to the future of radiological imaging. The ScienceDirect, PubMed, NCBI, Scopus, Elsevier and SAGE databases were utilized on September 2019 to search for publications containing information about DH in radiological imaging for the purpose of this report. The abstracts, which were discovered through this search, were re-considered for their applicability to the clinical outcomes associated with this procedure. Complete manuscripts were retrieved and considered for inclusion in this report if they featured data that evaluated the evidence on the role of DH with radiological imaging and assessed the possible impact on modern medicine, and if the manuscripts featured clinical practice. Only those studies published between 2000 and 2019 were included in the outcomes analysis; this was due to the tremendous evolution of DH that occurred at the beginning of the new millennium. With respect to the location of the research or the types of journals included in this study, all valid sources of experience were incorporated. There were no restrictions with respect to the country of origin of the publications, which led to a range of opinions and experiences being found that could serve as material for this report. Articles obtained through a refined search were surveyed individually to further explore their content.

\section{DH: Definition and Process}

Digital holograms are made up of holographic elements (hogels) rather than pixels or voxels. In contrast to pixels, which are normally used in photography, hogels contain 3D information from multiple perspectives. ${ }^{(11)} \mathrm{DH}$ is a two-step process. Step 1 involves recording the hologram, where the radiographic image is transformed into a photographic record. Step 2 consists of reconstruction, in which the hologram is transformed into a virtual image. The digital hologram created contains a grid of holographic pixels, also known as holopixels, or the aforementioned hogels. Over time, holopixels have been diminishing in scale, currently being around $0.8 \mathrm{~mm}$, and are now being reduced to $0.5 \mathrm{~mm}$ or even $0.25 \mathrm{~mm}$. Any kind of 3D medical dataset, such as CT and MR images, can be converted into a digital hologram. Such data can also be made available in the form of a physical scan of an object, a mathematical description of the images, molecular data or even a series of stills or videos. ${ }^{(10)}$

\section{$\underline{\text { DH in Medicine }}$}

Recently, Royal Philips and RealView Imaging reported on a clinical study demonstrating the feasibility of using 
innovative live 3D holographic visualization and interaction technology to guide minimally invasive structural heart disease procedures. ${ }^{(12)}$ RealView's visualization technology was used to display interactive, real-time 3D holographic images acquired by Philips' Interventional X-ray and cardiac ultrasound systems. Consequently, in addition to viewing the patient's heart on a 2D screen, interventional cardiologists were able to view detailed, dynamic 3D holographic images of the heart floating in free space during an interventional structural heart disease procedure, without having to use special eyewear. The physicians were also able to manipulate the projected $3 \mathrm{D}$ heart structures by literally touching the holographic volumes in front of them. Another area in which this technology may be useful is that of medical education. It may now be possible to deliver a presentation from a remote setting: A 3D, high-resolution, full-size image of the presenter could be projected onstage, onto a special podium, in front of a live audience thousands of kilometers away, thus allowing virtual lectures to be held. These technologies can also provide live simulations to aid students in learning a procedure. Other uses could include applications in telemedicine, 3D mapping technologies, remote guidance during emergencies, remote video conferencing, manufacturing, and myriad other uses. ${ }^{(1)}$ Regarding the DH industry, there are several companies that now produce true hologram systems that are specifically targeted towards the medical sector (Table 1). ${ }^{(1)}$

\section{Potential Applications of DH in Radiology}

There are three potential uses of holography in radiology: i) teaching radiologic anatomy, ii) PACS storage, and iii) image display. ${ }^{(10)}$ i) The medical hologram, particularly in radiology, presents a performance boost over traditional textbook images. The 3D nature of the image generated provides superior visual capabilities. ${ }^{(10)}$ The concept of optical components suggests that the $3 \mathrm{D}$ concept relies on the grouping of visual subcomponents based on textures, color grade and the linking of structure. ${ }^{(13)}$ This technique enables visualization of the intricate details of anatomical structures. Its use has also been documented in imaging the liver through ultrasound scans. Complex holograms containing information layers from images of the musculoskeletal, vascular, and nervous systems are now available. ${ }^{(10)}$

ii) There has been renewed interest in holography due to the recent propagation of $3 \mathrm{D}$ content and availability of $3 \mathrm{D}$ data from CT, MRI and ultrasound scanners, low-cost depth scanners, cinema/TV, and 3D printing. Holographic storage and retrieval systems can be looked upon as solutions to the demands being made for greater storage capacity, rapid retrieval, and smaller physical storage footprints. ${ }^{(10)}$ By adopting PACS, a turnaround has occurred in radiology productivity through the channeling of the radiology workflow. ${ }^{(14)}$ Holographic media can be used specifically for data storage due to their unique ability to store information in 3D, in contrast to standard optical disks, which store data only on the surface. In the near future, there is a high probability that this technology can eventually replace magnetic, single-layer storage with optical, 3D holograms. ${ }^{(15)}$ PACS currently requires excessive amounts of data storage, whereas holographic technology can provide up to 1000 times greater storage capacity than current methods..$^{(15,16)}$

With holography, we can record 1 million bits of data with a single flash of light. ${ }^{(17)}$ Comparative data suggests that

Table 1.

Digital holography industry in the medical sector ${ }^{(1)}$

\begin{tabular}{|c|c|c|c|}
\hline Company name & $\begin{array}{l}\text { Country of } \\
\text { origin }\end{array}$ & Product & Properties \\
\hline EchoPixel & USA & $\begin{array}{l}\text { True 3D Viewer } \\
(\mathrm{t} 3 \mathrm{~d})\end{array}$ & Converts 2D images to stereoscopic 3D images \\
\hline Zebra Imaging & USA & ZScape & $\begin{array}{l}\text { Allows the navigation of the entire body as well as sifting } \\
\text { through the skin layers, muscles, the cardiovascular system } \\
\text { and the skeleton }\end{array}$ \\
\hline $\begin{array}{l}\text { RealView } \\
\text { Imaging Ltd }\end{array}$ & Israel & 3D holograms (Holoscope) & $\begin{array}{l}\text { Employs digital light-shaping technology to create a unique } \\
\text { hyper-realistic experience for visualization of } 3 \mathrm{D} \text { medical } \\
\text { images }\end{array}$ \\
\hline $\begin{array}{l}\text { Mach7 } \\
\text { Technologies } \\
\text { Pte Ltd }\end{array}$ & Australia & Enterprise imaging platform & $\begin{array}{l}\text { Enterprise imaging platform provides the most robust, full- } \\
\text { functioning product set in the industry for PACS }\end{array}$ \\
\hline $\begin{array}{l}\text { Ovizio Imaging } \\
\text { System }\end{array}$ & Belgium & $\begin{array}{l}\text { Ovizio's microscopes (3D and 4D } \\
\text { uantitative microscopy) }\end{array}$ & $\begin{array}{l}\text { Real time 3D imaging with differential digital holography } \\
\text { microscopy }\end{array}$ \\
\hline Holoxica Ltd & $\begin{array}{l}\text { United } \\
\text { Kingdom }\end{array}$ & 3D digital holograms & $\begin{array}{l}\text { Facilitates the visualization of } 3 \mathrm{D} \text { data from every angle and } \\
\text { illustrate details that cannot be viewed in } 2 \mathrm{D} \text { images }\end{array}$ \\
\hline Lyncee Tec & Switzerland & $\begin{array}{l}\text { Digital holographic microscopes } \\
\text { with digital holographic camera }\end{array}$ & $\begin{array}{l}\text { Provides marker-free, non-invasive images of cells and allows } \\
\text { their characterization without perturbation }\end{array}$ \\
\hline Eon Reality & USA & $\begin{array}{l}\text { Virtual reality (VR) and } \\
\text { augmented reality (AR) software }\end{array}$ & $\begin{array}{l}\text { VR and AR products aimed at improving medical knowledge } \\
\text { transfer }\end{array}$ \\
\hline NanoLive SA & Switzerland & 3D cell explorer & $\begin{array}{l}\text { High precision holotomographic microscope, which facilitates } \\
\text { instantly being able to look inside living cells in 3D }\end{array}$ \\
\hline
\end{tabular}


the cost for new holographic media will probably be around $\$ 0.06-\$ 0.20$ per GB, as compared to data tape, which costs between $\$ 0.25$ and $\$ 1$, and videotape, which costs between $\$ 1$ and $\$ 3$ per GB. ${ }^{(15)}$

iii) The use of holographic displays in radiology is still in research, but they can soon find their way into commercial use and become economically viable. ${ }^{(18)} \mathrm{A}$ holographic screen can be created using a specially coated glass medium for the projection surface of a video projector, thereby creating a free-space display, as the image bearing surface appears very transparent. These views can be particularly advantageous in radiology, as computers will send the 3D CT data to a projector, which will beam it to a computer screen. Furthermore, 3D reconstruction of 2D images from sectional imaging can lead to detailed 3D models of body structures, which can then be holographically displayed. ${ }^{(10)}$ Holographic displays are presently insufficient with respect to giving good spatial resolution for general radiological interpretation. However, holography as a novel idea has been tried with very encouraging results in various other medical applications, such as echocardiography, guided invasive procedures, depiction of pelvic fractures, measurement of the motion of the tympanic membrane, depiction of bone fractures, and forensics. ${ }^{(19-21)}$ Recently developed display technology is now able to project 3D images in free space and give the clinician the ability to reach into the object to manipulate and apply markings to it. ${ }^{(10)}$

\section{DH: Limitations}

Holography requires relatively complicated methods to record holographic images, making it expensive and less accessible. Currently, holographic display technology lies far behind commercially available $2 \mathrm{D}$ and $3 \mathrm{D}$ displays. The most advanced volumetric displays currently have very poor spatial resolution and can display images at only about two frames per second. Holographic images are also inherently poor at visualizing subtle differences in tissue contrast. Storage solutions based on holography are facing significant challenges in bringing the technology into the market. A significant problem in holographic recording is the destructive readout. ${ }^{(10)}$ Such challenges have limited the adoption of DH in the field of radiology. In contrast, the potential impact of live holography on medical imaging is considerable, especially in the field of minimally invasive interventions, since these procedures demand a clear understanding of the patientspecific spatial anatomy. ${ }^{(22,23)}$

\section{DHI and its Role in the Future of Radiology}

Current radiology study images vary from $10 \mathrm{MB}$ to $90 \mathrm{MB}$ in size per test. A rough estimate shows that highresolution and specialized CT scans can occupy around $30 \mathrm{MB}$ of data per scan. Over the last decade, the quality of such investigations has undergone a change. Scans are now more focused, of higher resolution and are more detailed due to thinner sections and dynamic studies, which are possible due to the ability of both CT and MRI studies to create huge amounts of data. The current estimate suggests that for every 100,000 examinations performed, there will be the need to book approximately 3.1 terabytes of storage. ${ }^{(24)}$
Though holograms can create fascinating imagery, recording and presenting a visual object is not all they do. Holograms are capable of recording pure data- mountains of it. Holograms have the potential to store vast amounts of information. The current prototype systems store $4.4 \mathrm{~m}$ individual pages of information on a DVD-like disc. They also offer a unique form of long-term security. If someone makes an optical hologram of a page of information and then smashes it, for example, it can be reconstructed from any of the pieces. This makes holographic data storage extremely reliable. Unlike CDs and DVDs, which store their data on the disc's surface, holograms store data in 3D and those pages can overlap in storage space. ${ }^{(25)}$

Holography is on its way to revolutionizing medicine. It can be a tool for visualizing patient data, which will be of much use in training students and surgeons. Current systems like CT, MRI and ultrasound scans generate complex data using advanced imaging technology. This technology has the capability to produce full-color, computer-generated 3D holograms. For training and display, these holograms require no particular viewing devices or glasses. Students and doctors can simply look unhindered at the 3D images. These images can depict incredibly complex organs and systems of the body, like the brain, heart, liver, lungs, nerves and muscles. ${ }^{(25)}$

Due to the unique ability of DH to store data as a volumetric density and read it quickly, it is likely to become the storage medium of choice in the future.

\section{Conclusion}

DH will significantly disrupt $3 \mathrm{D}$ imaging as it has the potential to transform radiology and make it truly virtual. It can also influence every aspect of medical and clinical practice but in particular, it can be a game changer in radiology practice. Holographic imaging will provide radiologists and radiographers with the freedom to elevate the radiology workflow beyond high-resolution monitors, into free space. In addition, the complex issue of radiological image storage can be addressed with the use of holography. There are many questions remaining on how to exploit DHI fully in radiological practice. A plethora of clinical trials is currently investigating the implementation of $3 \mathrm{D} \mathrm{DHI}$ and its significance in the future of radiology, and on patients.

\section{Acknowledgments}

This publication was supported by the Deanship of Scientific Research at Prince Sattam bin Abdulaziz University, Alkharj, Saudi Arabia.

\section{Competing Interests} interests.

The authors declare that they have no competing

\section{References}

1. Mishra S. Hologram the future of medicine - From Star Wars to clinical imaging. Indian Heart J. 2017;69(4):566-567. doi:10.1016/j.ihj.2017.07.017 
2. Gabor D. A new microscopic principle. Nature. 1948;161(4098):777. doi:10.1038/161777a0

3. Held RT, Hui TT. A guide to stereoscopic 3D displays in medicine. Acad Radiol. 2011;18(8):1035-1048. doi:10.1016/j. acra.2011.04.005

4. Osten W, Faridian A, Gao P, Körner K, Naik D, Pedrini $\mathrm{G}$, et al. Recent advances in digital holography [invited]. Appl Opt. 2014;53(27):G44-G63. doi:10.1364/AO.53.000G44

5. Yu X, Hong J, Liu C, Cross M, Haynie DT, Kim MK. Fourdimensional motility tracking of biological cells by digital holographic microscopy. J Biomed Opt. 2014;19(4):045001. doi:10.1117/1.JBO.19.4.045001

6. Skolnick AA. New holographic process provides noninvasive, 3-D anatomic views. JAMA. 1994;271(1):5-8.

7. Hunziker PR, Smith S, Scherrer-Crosbie M, Liel-Cohen $\mathrm{N}$, Levine RA, Nesbitt R, et al. Dynamic holographic imaging of the beating human heart. Circulation. 1999;99(5):1-6. doi:10.1161/01.cir.99.5.1

8. Addetia K, Lang RM. The future has arrived. Are we ready? Eur Heart J Cardiovasc Imaging. 2016;17(8):850-851. doi:10.1093/ehjci/jew111

9. Bruckheimer E, Rotschild C, Dagan T, Amir G, Kaufman A, Gelman S, Birk E. Computer-generated real-time digital holography: first time use in clinical medical imaging. Eur Heart J Cardiovasc Imaging. 2016;17(8):845-849. doi:10.1093/ ehjci/jew087

10. Kharat AT, Kalra R, Shah A, Singh A. Exploring the potential use of holographic imaging in radiology. Appl Radiol. 2016;45(12):19-22.

11. Plesniak W, Pappu R, Underkoffler J, Lucente M, St.-Hilaire P. In: Benton SA, Bove VM, editors. Holographic Imaging. $1^{\text {st }}$ ed. Hoboken, New Jersey: Wiley-Interscience; 2008:207-229.

12. Philips and RealView Imaging Conclude World's First Study to Evaluate Live 3D Holographic Imaging in Interventional Cardiology. https://www.meddeviceonline. com/doc/philips-realview-imaging-holographic-imagingcardiology-0001.

13. Buhmann JM, Malik J, Perona P. Image recognition: visual grouping, recognition, and learning. Proc Natl Acad Sci U S A. 1999;96(25):14203-14204. doi:10.1073/pnas.96.25.14203 14. Lepanto L, Paré G, Aubry D, Robillard P, Lesage J. Impact of PACS on dictation turnaround time and productivity. J Digit
Imaging. 2006;19(1):92-97. doi:10.1007/s10278-005-9245-8 15. Colang J, Johnston J. PACS storage technology update: holographic storage. Radiol Manage. 2006;28(3):38-49.

16. Nagy P, Farmer J. Demystifying data storage: archiving options for PACS. Appl Radiol. 2004;33(5):18-22.

17. Liu Y, Kitamura K, Ravi G, Takekawa S, Nakamura M. Growth and two-color holographic storage properties of Mn-doped lithium niobate crystals with varying $\mathrm{Li} / \mathrm{Nb}$ ratio. Journal of Applied Physics. 2004;96(11):5996-6001. doi: $10.1063 / 1.1812824$

18. Bove VM. Display holography's digital second act. Proceedings of the IEEE. 2012;100(4):918-928. doi: 10.1109/ JPROC.2011.2182071.

19. Galeotti JM, Siegel M, Stetten G. Real-time tomographic holography for augmented reality. Opt Lett. 2010;35(14):23522354. doi:10.1364/OL.35.002352

20. Del Socorro Hernández-Montes M, Furlong C, Rosowski JJ, Hulli N, Harrington E, Cheng JT, Ravicz ME, Santoyo FM. Optoelectronic holographic otoscope for measurement of nano-displacements in tympanic membranes. J Biomed Opt. 2009;14(3):034023. doi:10.1117/1.3153898

21. Biwasaka H, Saigusa K, Aoki Y. The applicability of holography in forensic identification: a fusion of the traditional optical technique and digital technique. J Forensic Sci. 2005;50(2):393-399.

22. Lee SL, Lerotic M, Vitiello V, Giannarou S, Kwok KW, Visentini-Scarzanella M, Yank GZ. From medical images to minimally invasive intervention: Computer assistance for robotic surgery. Comput Med Imaging Graph. 2010;34(1):3345. doi:10.1016/j.compmedimag.2009.07.007

23. Soler L, Nicolau S, Pessaux P, Mutter D, Marescaux J. Real-time 3D image reconstruction guidance in liver resection surgery. Hepatobiliary Surg Nutr. 2014;3(2):73-81. doi:10.3978/j.issn.2304-3881.2014.02.03

24. Frejlich J, de Oliveira I, Arizmendi L, Carrascosa M. Fixed holograms in iron-doped lithium niobate: simultaneous self-stabilized recording and compensation. Appl Opt. 2007;46(2):227-233. doi:10.1364/ao.46.000227

25. Kucirkova A. What Can We Expect from Hologram Technology in the Future? Published Jul 17, 2018. Available from: https:/www.nasdaq.com/articles/what-can-we-expecthologram-technology-future-2018-07-17. 